\title{
The Role of the Nose in Obstructive Sleep Apnea: A Short Review
}

\author{
Die Rolle der Nasenatmung bei obstruktiver Schlafapnoe
}

Author

Institution

\section{A. Valipour}

Department of Respiratory and Critical Care Medicine, Ludwig-Boltzmann-Institute for COPD and Respiratory Epidemiology, Otto-Wagner-Hospital, Vienna, Austria received 6.3 .2014

accepted 19.3.2014

\section{Bibliography}

DOI http://dx.doi.org/

10.1055/s-0034-1365488

Published online: 13.5.2014

Pneumologie 2014; 68: 397-400

(c) Georg Thieme Verlag KG

Stuttgart · New York

ISSN 0934-8387

\section{Corresponding author Arschang Valipour}

Department of Respiratory and Critical Care Medicine, LudwigBoltzmann-Institute for COPD and Respiratory Epidemiology, Otto-Wagner-Hospital, Vienna, Austria

Sanatoriumsstrasse 2

1140 Vienna

Austria

arschang.valipour@wienkav.at

\section{Abstract $\nabla$}

A variety of causes may result in nasal obstruction including allergic and non-allergic rhinitis, anatomic abnormalities, and postoperative nasal packing. There is both an epidemiological and clinical relationship between nasal obstruction and sleep disordered breathing. Subsequently, improving nasal patency via surgical or non-surgical means is expected to relieve sleep disordered breathing. The present review will discuss and review some of the literature related to the pathophysiological interaction of the human nose and sleep disordered breathing and provide a summary of the effects of related intervention trials.

\section{Impact of nasal obstruction}

Obstructive sleep apnea syndrome (OSAS) is a common condition with a prevalence of $2-4 \%$ of men and $1-2 \%$ of women. A variety of risk factors have been reported for OSAS, including obesity, male gender, craniofacial abnormalities, and/or nasal obstruction. The latter has been supported by a number of observational and cross-sectional studies that have demonstrated a relationship between nasal obstruction and obstructive sleep apnea [1]. Nasal obstruction may result from allergic and non-allergic rhinitis, anatomic abnormalities, postoperative nasal packing, and/or experimental nasal occlusion. In a populationbased study, Young et al. [2] previously suggested a relationship between frequent symptoms of nasal obstruction (5 or more nights a month) and reports of habitual snoring, chronic excessive daytime sleepiness, or chronic nonrestorative sleep. Subsequently, improving nasal patency via

\section{Zusammenfassung \\ $\nabla$}

Eine Reihe von Ursachen können zu einer nasalen Obstruktion führen, beispielsweise die allergische und nicht-allergische Rhinitis, anatomische Abnormitäten und postoperative Nasentamponaden. Es existiert ein epidemiologischer wie auch klinischer Zusammenhang zwischen nasaler Obstruktion und schlafbezogenen Atmungsstörungen. Folglich kann man erwarten, dass eine Verbesserung der nasalen Atmung mittels chirurgischer oder nicht-chirurgischer Maßnahmen zu einer Normalisierung der schlafbezogenen Atmungsstörungen führt. In dieser Übersicht wird die Literatur zu den pathophysiologischen $\mathrm{Zu}$ sammenhängen zwischen der menschlichen Nase und den schlafbezogenen Atmungsstörungen diskutiert und eine Zusammenfassung der klinischen Evidenz therapeutischer Interventionen präsentiert.

surgical or non-surgical means is expected to relieve sleep disordered breathing. We will discuss and review some of the literature related to the pathophysiological interaction of the human nose on sleep and sleep disordered breathing and provide a summary of the effects of related intervention trials. Some of the therapeutic intervention trials presented in this review have been summarized in a 2011 Task Force Report of the European Respiratory Society [3].

\section{Nasal airflow and breathing}

Breathing through the human nose appears to have an effect on both ventilation and upper airway muscle tone. In an experimental study, McNicholas and co-workers [4] previously demonstrated increased ventilation during nasal ventilation compared to mouth breathing. Using a different approach to this question, White et al [5] investigated the effects of local nasal anesthesia on ventilation in 10 normal men during sleep. Each sub- 
Table 1 Nasal obstruction and OSA. Pathophysiological impact of nasal obstruction in the development of obstructive sleep apnea.

\begin{tabular}{ll}
$\begin{array}{l}\text { Breathing through the human } \\
\text { nose results in }\end{array}$ & $\begin{array}{l}\text { Partial or complete nasal } \\
\text { occlusion results in }\end{array}$ \\
\hline - ventilation & - oral breathing periods \\
- upper airway dilator & - upper airway resistance \\
- muscle activity & - obstructive apnea events \\
- upper airway stability & - arousals \\
\hline
\end{tabular}

ject underwent polysomnographic monitoring of sleep stages, breathing patterns, respiratory effort, and arterial oxygen saturation. The application of lidocaine (plus a decongestant) resulted in a fourfold increase in the number of both central and obstructive apneas in the study population compared to the placebo night. These findings suggest a stimulating effect of nasal airflow on central respiratory drive and upper airway stability ( Table 1 ). The latter has been supported by a study from Basner RC et al. [6]. The authors investigated eight healthy males (age range 21-38 yrs) during successive trials of voluntary nose breathing, mouth breathing, and mouth breathing with the nose occluded. They measured genioglossus electromyographic activity, alae nasi and diaphragm EMG activity, and minute ventilation. While they observed no effect of breathing route on minute ventilation or diaphragm activity, nasal ventilation was associated with higher upper airway dilator muscle activity than breathing through the mouth. Fitzpatrick et al. [7] confirmed these observations by demonstrating evidence of increased upper airway resistance during sleep while breathing through the mouth when compared to nasal breathing. The authors performed a randomised, crossover study to compare upper airway resistance during sleep of nasal and oral breathing in 12 healthy non-obese subjects (age $30 \pm 4$ yrs) with normal nasal resistance. During wakefulness upper airway resistance values were similar between nasal and oral breathing in both lateral and supine position. In contrast, there was evidence of increased upper airway resistance, an increased number of apneas and hypopneas, and lower total sleep time with mouth breathing during sleep. In order to investigate whether these findings may also be applicable to patients with obstructive sleep apnea, Kotsourelakis et al. [8] investigated the breathing route of 28 patients with OSAS (AHI 26 $\pm 5 / \mathrm{hr}$ ) and 13 snorers (AHI $1.3 \pm 0.3 / \mathrm{hr}$ ) without sleep apnea during overnight polysomnography using both a nasal cannula/pressure transducer and an oral thermistor. Subjects were free from nasal pathology and had normal nasal resistance on rhinomanometric measurements in seated and supine position while awake. During sleep, nasal breathing epochs were more frequently observed in snorers than in apneics, whereas the inverse was true for oral and oro-nasal breathing epochs. Oral breathing epochs were furthermore positively related with $\mathrm{AHI}$, mean and maximum duration of apnea/hypopnea, and were inversely related to average and minimum oxygen saturation. Thus, in summary these findings suggest a protective effect of nasal breathing on upper airway stability. The current literature furthermore indicates that patients with OSAS are more likely to breath through the high resistance pathway of the mouth, thereby promoting more negative intraluminal pressure in the pharynx predisposing to pharyngeal occlusion and thus obstructive apnea events.
The effects of nasal occlusion on sleep and breathing Olsen et al. [9] studied the effects of experimental nasal obstruction on sleep and breathing in eight male volunteers (30 to 50 years of age). None of the subjects was obese; furthermore none of the subjects reported sleep problems or difficulties in breathing through the nose. The subjects were randomised into two groups. One night subjects were studied with the nose open and a second night with the nose obstructed using a small amount of cotton and Vaseline placed inside the nasal vestibule and covered with an adhesive tape. The authors demonstrated an increased number of arousals, more frequent sleep stage changes, and a significant increase in the number of obstructive apneas and hypopneas during sleep associated with nasal occlusion. Furthermore, the subjects complained about dry mouth, sore throat, frequent awakenings, and restlessness following the night of experimental nasal obstruction. Using a similar study design, Zwillich et al. [10] investigated the effects of nasal obstruction on polysomnographically derived parameters in ten normal men between the age of 25 and 45. Nasal occlusion was accomplished using an inflatable balloon that was placed into each nostril. The subjects slept during 3 consecutive nights with all instruments in place, including one standard acclimatisation night, one night with the balloon inflated and another night with the balloon deflated. The order of the obstructed and non-obstructed night was randomised. Consistent with the findings above, the authors observed more frequent arousals, decreased slow wave sleep, and a significantly increased number of (predominantly central) apneas per night during obstructed sleep (total number of apneas $88 \pm 34$ ) than non-obstructed sleep (total number of apneas $34 \pm 19$ ). Lavie and colleagues [11] extended these findings, as they used an experimental design that included unilateral or bilateral nasal occlusion. They studied 5 men and 5 women between 20 and 27 years of age on four nights (no occlusion, either left or right, or bilateral occlusion of the nostrils with an adhesive tape). Both partial and complete nasal occlusion was associated with an increased number of apneas and hypopneas, as well as disturbed sleep architecture, with the respective study findings being more pronounced with bilateral nasal occlusion. In a subsequent report, Lavie [12] studied the effects of nasal obstruction in 6 sons of sleep apnea patients (mean age 21 yrs, BMI not reported). Nasal occlusion resulted in a marked increase in the total number of apneas from 18 to 303 per night in this group, an observation that was much weaker in matched controls. This report provided early evidence of potential inheritability of upper airway obstruction during sleep.

Nasal obstruction may also be a result of bilateral nasal packing followig nasal surgery. Results from earlier studies monitoring peri- and postoperative oxygen saturation in patients who received total nasal packs after septoplasty, revealed evidence of significant postoperative nocturnal hypoxemia. Regli et al. [13] performed a case-control trial using polygraphy prior to and after nasal packing in a group of 15 patients with OSAS (median AHI 14/hr, 80\% male, median age 50 yrs, median ESS 13, median BMI $31 \mathrm{~kg} / \mathrm{m}^{2}$ ) and another group of 25 individuals without OSAS (median AHI 11/hr, 76\% male, median age 46 yrs, median ESS 5, median BMI $26 \mathrm{~kg} / \mathrm{m}^{2}$ ). The authors observed a significant increase in postoperative AHI in both groups (median AHI 37/hr in non-OSAS and 39/hr in OSAS-group) and an increase in the oxygen desaturation index in patients without sleep apnea. Most recently, Friedman et al. [14] tested the effect of postoperative nasal packing on sleep parameters between patients with mild obstructive sleep apnea and those with moderate to severe ob- 
structive sleep apnea. All subjects underwent polysomnography testing within 30 days prior to surgery. Patients underwent nasal surgery and received postoperative nasal packing, and overnight sleep testing was repeated on the first postoperative night with nasal packing in place. Interestingly, postoperative nasal packing aggravated measures of sleep apnea in patients with mild but not in patients with moderate to severe obstructive sleep apnea. In summary there is evidence of both inducing and worsening of sleep disordered breathing in association with nasal occlusion.

\section{Effects of nasal decongestion or intranasal steroids on sleep and OSA \\ $\nabla$}

A particularly frequent cause of nasal obstruction is allergic rhinitis, either seasonal or perennial. A number of reports suggested worsening of subjective sleep quality and quality of life measures in both adults and children with allergic rhinitis [15]. In fact, a recent study demonstrated that perennial rhinitis was associated with an increased probability for difficulties maintaining sleep, early morning awakenings and daytime sleepiness [16]. Vice versa, Canova and co-workers [17] demonstrated an increased prevalence of perennial allergic rhinitis in patients with OSA (11\%) compared to case-controls (2,3\%). At least two pathways may contribute to the development or worsening of sleep apnea due to allergic rhinitis. First, upper airway allergy causes oedema and mucus secretion in the nasal mucosa, which may predispose to the development of partial or complete upper airway obstruction during sleep via increasing upper airway resistance. Second, inflammation of the nasal mucosa may contribute to the development of adenotonsillar hypertrophy, one of the most frequently observed abnormalities in children with OSA. Intranasal corticosteroids are commonly used to treat rhinitis. Besides their effects on IgE mediated inflammation, topical nasal steroids may also exert direct lympholytic and/or anti-inflammatory effects [18]. Improving nasal obstruction symptoms and reducing adenoid size with intranasal corticosteroids may thus influence the anatomic component by decreasing upper airway resistance at the nasal, adenoidal, and/or tonsillar levels.

The scientific evidence regarding the effects of nasal steroids is predominantly driven by studies performed in children and only little evidence exists regarding the impact on polysomnographically diagnosed sleep parameters in adult patients with moderate OSA and co-existing (perennial or seasonal) rhinitis. Most of the children included in these reports had evidence of co-existing rhinitis and/or adenotonsillar hypertrophy. Overall, these reports indicate significant treatment associated improvements in the AHI (mean pre-treatment AHI range 3.7-11 vs. treatment associated mean AHI range $0.3-6$ ) and some of the reports showed significant improvements in oxygenation indices and improved sleep quality [3]. The data are, however, inconsistent with respect to sleep architecture and reduction in adenoidal size. The effects of intranasal corticosteroid application on polysomnographically diagnosed sleep parameters in adult patients with OSAS and associated rhinitis is limited. In a 4 week, randomized, doubleblind, placebo-controlled, cross-over trial of nasal fluticasone in 13 patients with moderate OSA (mean AHI 26/hr), Kiely and coworkers [19] demonstrated a modest but significant decrease in AHI (median -6.5/hr AHI reduction) and an improvement in nasal airflow resistance, however, no significant improvements in oxygenation indices, sleep quality or snoring noise. More recently, Kotsourelakis et al. [20] investigated the effects of a combina- tion of a nasal decongestant with corticosteroids on the breathing route pattern and sleep disordered breathing in 21 patients with OSA and mean AHI 31/hr. Using a randomized, placebo-controlled, cross-over trial the authors were able to demonstrate a mean decrease in the $\mathrm{AHI}$ by $21 \%$. The latter was predominantly due to an increase in nasal breathing epochs.

\section{Nasal surgery on obstructive sleep apnea \\ $\nabla$}

The majority of reports on nasal surgery as a single-intervention in OSA have been published either as case-series or retrospective analyses [3]. One report [21] was conducted as a prospective, randomised, sham-controlled trial. The most frequently observed pathologic finding in the preoperative ENT examination was nasal obstruction due to deviated nasal septum. Accordingly, septal surgery (submucosal resection with or without turbinectomy) was the most frequently applied surgical technique in these reports. All the studies that have performed rhinomanometry reported significant postoperative improvements in total nasal resistance, indicating postoperative improvements in nasal airway patency in patients.

Overall, the large majority of studies reported no significant changes in the AHI (RDI) (mean preoperative AHI range 14-56/ hr vs. mean postoperative AHI range $15-48 / \mathrm{hr}$ ), including the above mentioned study [21], which was conducted as a randomised sham-controlled intervention trial in a matched population. One study [22] reported a significant improvement in AHI and sleep architecture in patients with normal preoperative cephalometric measurements, but no beneficial effect in a group of patients with abnormal cephalometric measurements prior to surgery, suggesting that the success of nasal surgery may be predictable by preoperative anatomic findings. Despite the lack of beneficial effects with respect to the AHI, there have been improvements in either sleepiness scales or daytime energy levels, and a reduction in therapeutic CPAP pressure required to alleviate OSA in some of the studies. Thus on the basis of the above mentioned findings, nasal surgery as a single intervention cannot be recommended for treatment of obstructive sleep apnea [3].

\section{Conclusions}

The nasal breathing route is important to upper airway stability. Studies of nasal obstruction demonstrated initiation or worsening of sleep disordered breathing. Relief of nasal obstruction via intranasal decongestants and/or steroids, or via nasal surgery have been shown to attenuate sleep disordered breathing.

\section{Conflict of interest \\ $\nabla$}

The author has no conflict of interest. 


\section{References}

1 Kohler M, Bloch KE, Stradling JR. The role of the nose in the pathogenesis of obstructive sleep apnoea and snoring. Eur Respir J 2007; 30: $1208-1215$

2 Young T, Finn L, Kim H. Nasal obstruction as a risk factor for sleep-disordered breathing. The University of Wisconsin Sleep and Respiratory Research Group. J Allergy Clin Immunol 1997; 99: 757-762

3 Randerath WJ, Verbraecken J, Andreas $S$ et al. European Respiratory Society task force on non-CPAP therapies in sleep apnoea. Non-CPAP therapies in obstructive sleep apnoea. Eur Respir J 2011; 37: 10001028

4 McNicholas WT, Coffey M, Boyle T. Effects of nasal airflow on breathing during sleep in normal humans. Am Rev Respir Dis 1993; 147: 620623

5 White DP, Cadieux RJ, Lombard RM et al. The effects of nasal anesthesia on breathing during sleep. Am Rev Respir Dis 1985; 132: 972 - 975

6 Basner RC, Simon PM, Schwartzstein RM et al. Breathing route influences upper airway muscle activity in awake normal adults. J Appl Physiol 1989; 66: $1766-1771$

7 Fitzpatrick MF, McLean H, Urton AM et al. Effect of nasal or oral breathing route on upper airway resistance during sleep. Eur Respir J 2003; 22: $827-832$

8 Koutsourelakis I, Vagiakis E, Roussos C et al. Obstructive sleep apnoea and oral breathing in patients free of nasal obstruction. Eur Respir J 2006; 28: $1222-1228$

9 Olsen KD, Kern EB, Westbrook PR. Sleep and breathing disturbance secondary to nasal obstruction. Otolaryngol Head Neck Surg 1981; 89: $804-810$

10 Zwillich CW, Pickett C, Hanson FN et al. Disturbed sleep and prolonged apnea during nasal obstruction in normal men. Am Rev Respir Dis 1981; $124: 158-160$

11 Lavie P, Fischel N, Zomer J et al. The effects of partial and complete mechanical occlusion of the nasal passages on sleep structure and breathing in sleep. Acta Otolaryngol 1983; 95: 161 -166
12 Lavie P, Rubin AE. Effects of nasal occlusion on respiration in sleep. Evidence of inheritability of sleep apnea proneness. Acta Otolaryngol 1984; 97: $127-130$

13 Regli A, von Ungern-Sternberg BS, Strobel WM et al. The impact of postoperative nasal packing on sleep-disordered breathing and nocturnal oxygen saturation in patients with obstructive sleep apnea syndrome. Anesth Analg 2006; 102: 615-620

14 Friedman $M$, Maley $A$, Kelley $K$ et al. Impact of Nasal Obstruction on Obstructive Sleep Apnea. Otolaryngol Head Neck Surg 2011; 144: $1000-1004$

15 Muliol J, Maurer M, Bousquet J. Sleep and allergic rhinitis. J Investig Allergol Clin Immunol 2008; 18: 415-419

16 Hellgren J, Omenaas E, Gíslason T et al. RHINE Study Group, North Europe. Perennial non-infectious rhinitis - an independent risk factor for sleep disturbances in Asthma. . Respir Med 2007; 101: 1015-1020

17 Canova CR, Downs SH, Knoblauch A et al. Increased prevalence of perennial allergic rhinitis in patients with obstructive sleep apnea. Respiration 2004; 71: $138-143$

18 Kheirandish-Gozal L, Serpero LD, Dayyat E et al. Corticosteroids suppress in vitro tonsillar proliferation in children with obstructive sleep apnoea. Eur Respir J 2009; 33: 1077-1084

19 Kiely JL, Nolan P, McNicholas WT. Intranasal corticosteroid therapy for obstructive sleep apnoea in patients with co-existing rhinitis. Thorax 2004; 59: 50-55

20 Koutsourelakis I, Minaritzoglou A, Zakynthinos G et al. The effect of nasal tramazoline with dexamethasone in obstructive sleep apnoea patients. Eur Respir J 2013; 42: 1055-1063

21 Koutsourelakis I, Georgoulopoulos G, Perraki E et al. Randomised trial of nasal surgery for fixed nasal obstruction in obstructive sleep apnoea. Eur Respir J. 2008; 31: 110-117

22 Sériès F, St PierreS, Carrier G. Surgical correction of nasal obstruction in the treatment of mild sleep apnoea: importance of cephalometry in predicting outcome. Thorax 1993; 48: 360-363 